\title{
Combined Intervention of Medium-Chain Triacylglycerol Diet and Exercise Reduces Body Fat Mass and Enhances Energy Expenditure in Rats
}

\author{
Katsuhiko OOYAmA $^{1}$, Jian Wu ${ }^{1,2}$, Naohisa NOSAKA ${ }^{1}$, Toshiaki AOYAMA ${ }^{1}$ and Michio KASAI ${ }^{1}$ \\ ${ }^{1}$ Division of Healthcare Science, Central Research Laboratory, The Nisshin OilliO Group, Ltd., 1 Shinmei-cho, \\ Yokosuka, Kanagawa 239-0832, Japan \\ ${ }^{2}$ National Institute of Health and Nutrition, 1-23-1 Toyama, Shinjuku-ku, Tokyo 162-8636, Japan
}

(Received September 4, 2007)

\begin{abstract}
Summary Previous studies indicated that a medium-chain triacylglycerol (MCT) diet could inhibit body fat accumulation. It is also well established that exercise can reduce fat mass. However, the effects of a combination of MCT diet and exercise on reduction of fat mass have not been studied. Here we examined whether MCT diet and exercise intervention exert cooperative effects on body composition. Rats were assigned to 4 groups: 1 . LCT diet, control (LCT-C); 2. MCT diet, control (MCT-C); 3. LCT diet, exercise (LCT-E); 4. MCT diet, exercise (MCT-E). After the 6-wk intervention, visceral fat mass was measured by CT scan and dissection, and energy expenditure was estimated for $24 \mathrm{~h}$. The value of the visceral fat mass showed a significant correlation between CT scan and dissection $(r=0.995, p<0.001)$. Visceral fat mass in the MCT-C group was lower than that in the LCT-C group. Furthermore, the fat-lowering effects were greater in the MCT-E group than that in either intervention alone. Thus significant effects of the MCT diet and exercise on the reduction of visceral fat mass were observed. Energy expenditure was significantly higher in the MCT-E group than in the other groups. Our present findings suggest that combined intervention of MCT diet and exercise has an additive effect on reduction of visceral and subcutaneous fat accumulation, and that this effect may be partially related to increased energy expenditure. However, future studies are necessary to define the relationship between energy expenditure and fat mass accumulation.
\end{abstract}

Key Words medium-chain triacylglycerols, fat mass, exercise, energy expenditure, CT scan

Medium-chain triacylglycerol (MCT) is edible oil composed of $\mathrm{C} 8$ and $\mathrm{C} 10$ saturated fatty acids. Compared with long-chain triacylglycerol (LCT), MCT has several unique physiological and biological characteristics (1, 2). Medium-chain fatty acids (MCFA) are absorbed through the portal system without resynthesis of triacylglycerol in intestinal cells and are predominantly subjected to $\beta$-oxidation in the liver. Only a small proportion of such fatty acids is stored as fat (3). Consequently, MCT are easily oxidized, because their intramitochondrial transport does not require carnitine palmitoyltransferase (CPT), the rate-limiting enzyme of mitochondrial $\beta$-oxidation. Therefore, the digestion, absorption and metabolism of MCT differ markedly from those of LCT (4). Recently it has been reported that a MCT diet decreases the deposition of fat in adipose tissue in humans and rats $(5,6)$.

The accumulation of visceral fat, independent of total body fat, is associated with the development of metabolic syndrome, characterized by dyslipidemia, hypertension, glucose intolerance, and hyperinsulinemia (79). Therefore, it is important to reduce the visceral fat mass in order to prevent metabolic syndrome. It is well

E-mail: k-ooyama@nisshin-oillio.com established that exercise training is beneficial for control of body weight and fat mass (10-12), and that endurance exercise training can enhance adipocyte lipolysis in animals and humans $(13,14)$. Fushiki et al. (15) report an increase of swimming endurance capacity of mice with the combination of MCT diet and exhaustion exercise. However, the combined effects of MCT diet with moderate (aerobic) exercise on reduction of fat mass have not been well-defined. In this study, we examined the combined effects of a MCT diet and running exercise on visceral and subcutaneous fat mass in rats by using a CT scan. We also compared the difference between the methods of measurement of the visceral fat: by CT scan and by dissection.

\section{MATERIALS AND METHODS}

Materials. MCT (consisting of octanoate and decanoate) and LCT (rapeseed oil as a common edible oil) were prepared by The Nisshin OilliO Group, Ltd. (Tokyo, Japan). Their fatty acid compositions (Table 1) were measured by gas chromatography (6890 series; Agilent Technologies, Palo Alto, California, USA) with a capillary column (SP2340; Supelco, Bellefonte, Pennsylvania, USA).

Animals and diets. All animals were treated in accor- 
Table 1. Fatty acid compositions of test oils. ${ }^{1}$

\begin{tabular}{lcc}
\hline \multicolumn{1}{c}{ Fatty acid } & $\mathrm{LCT}^{3}$ & $\mathrm{MCT}^{4}$ \\
\hline & $(\mathrm{g} / 100 \mathrm{~g}$ total fatty acids $)$ \\
8:0 & $\mathrm{ND}$ & 74.4 \\
$10: 0$ & $\mathrm{ND}$ & 25.6 \\
$16: 0$ & 4.2 & $\mathrm{ND}$ \\
$18: 0$ & 2.0 & $\mathrm{ND}$ \\
$18: 1$ & 59.6 & $\mathrm{ND}$ \\
$18: 2(n-6)$ & 20.4 & $\mathrm{ND}$ \\
$18: 3(n-3)$ & 10.2 & $\mathrm{ND}$ \\
Others & 3.6 & $\mathrm{ND}$ \\
\hline
\end{tabular}

${ }^{1}$ Values determined by gas chromatography.

${ }^{2}$ Number of carbon atoms:number of double bonds.

${ }^{3}$ Rapeseed oil.

${ }^{4}$ Medium chain triacylglycerols.

${ }^{5}$ Not detected.

dance with the guidelines for the care and use of laboratory animals (Notification of the Prime Minister's Office in Japan). Five-week-old male Wistar rats were purchased from SLC (Hamamatsu, Japan). They were housed individually in stainless steel wire-bottom cages in a room maintained at $22-24^{\circ} \mathrm{C}$ with a 12 -h lightdark cycle (dark period: 08:00-20:00). The food and body weight (BW) were measured in the dark-period before the running exercise. After a week of adapting to the environment, the rats were divided into the following four BW-matched groups with six rats in each group: 1. LCT diet control group (LCT-C), 2. MCT diet control group (MCT-C), 3. LCT diet and exercise group (LCT-E) and 4. MCT diet and exercise group (MCT-E). The LCT diet contained the following ingredients (g/ $\mathrm{kg}$ ): cornstarch, 479.5 (8.0 MJ); casein, 200 (3.3 MJ); sucrose, 100 (1.7 MJ); LCT, 120 (4.5 MJ); AIN-93G mineral mix, 35; AIN-93G vitamin mix (16), 10; cellulose, 50; L-cystine, 3; choline bitartrate, 2.5; and TBHQ, 0.045 (total energy:17.5 MJ). The MCT diet contained the following ingredients $(\mathrm{g} / \mathrm{kg})$ : cornstarch, 504.5 (8.4 MJ); casein, 200 (3.3 MJ); sucrose, 100 (1.7 MJ); LCT, 20 (0.8 MJ); MCT, 100 (3.3 MJ); AIN-93G mineral mix, 35; AIN-93G vitamin mix, 10; cellulose, 25; L-cystine, 3; choline bitartrate, 2.5; and TBHQ, 0.045 (total energy: $17.5 \mathrm{MJ}$ ). We matched the lipid content with equal weight, so we corrected cornstarch and cellulose contents to match total energy. The feeding was done every day and BW was measured every week. Each group of rats were managed by the pair-fed control method. The pair-fed rats were fed an amount of food that was equal to the average amount of food consumed by the LCT-E group or by the MCT-E group in the previous feed.

Exercise protocol. The exercise regimen consisted of running $5 \mathrm{~d} / \mathrm{wk}$ on a flatbed treadmill for $45 \mathrm{~min} / \mathrm{d}$ at $20 \mathrm{~m} / \mathrm{min}$. The running exercise was performed between 10:00 and 12:00. To acclimate the rats to running exercise, all of the rats were run on a treadmill (Natsume Corp., Tokyo, Japan) for $3 \mathrm{~d}$ before the experiment. The speed of the treadmill and the duration of
A) A CT scan image of a subject.

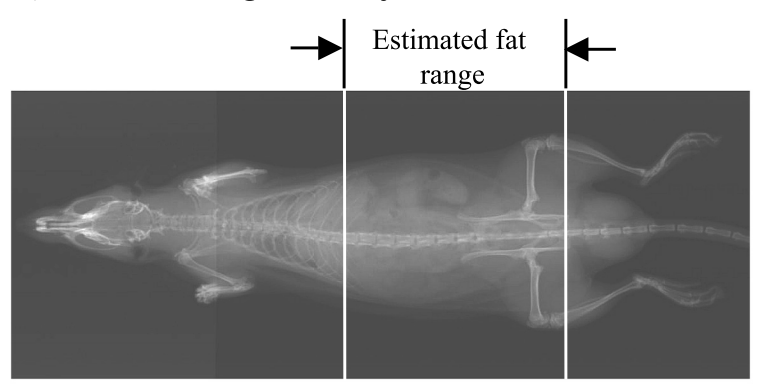

B) A CT-scan slice at a level $40 \mathrm{~mm}$ below the diaphragm.

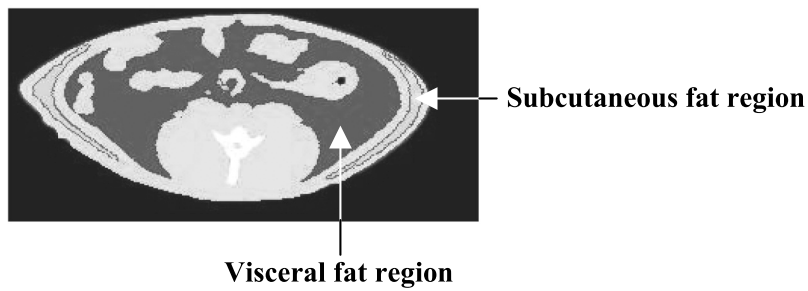

Fig. 1. CT scan image. (A) A CT scan image of a subject. Estimation of fat volumes (visceral and subcutaneous fat mass) were conducted in the abdominal region from the diaphragm to the bottom of the abdominal cavity at 2-mm CT slice intervals. (B) A CT scan slice at $40 \mathrm{~mm}$ below the diaphragm. The inner area is the visceral fat region, and the outer regions on each side are subcutaneous fat regions.

each running session were gradually increased from $15 \mathrm{~m} / \mathrm{min}$ for $15 \mathrm{~min}$ to $20 \mathrm{~m} / \mathrm{min}$ for $45 \mathrm{~min}$. Thus, this running intensity and duration $(20 \mathrm{~m} / \mathrm{min}$ for $45 \mathrm{~min}$ ) were done by the exercise groups from the beginning to the end of the study. The running regimen did not involve any form of electrical shock device. If rats stopped running they were nudged with a soft brush to encourage them to continue.

Energy expenditure. All of the rats were measured in the metabolic chamber in the 5th or 6th week of study. The rats in the exercise groups were measured on the weekend (no exercise training day). Oxygen consumption and carbon dioxide production were measured every $3 \mathrm{~min}$ for $24 \mathrm{~h}$ under resting conditions using a metabolism measuring system for small animals (MK5000R; Muromachi Kikai, Tokyo, Japan). A reference gas $\left(5 \% \mathrm{CO}_{2}, 95 \% \mathrm{O}_{2}\right)$ was used to calibrate this system. $\mathrm{VO}_{2}(\mathrm{~mL} / \mathrm{min}), \mathrm{VCO}_{2}(\mathrm{~mL} / \mathrm{min}), \mathrm{RQ}$, gas pressures, airflow, cage temperature, ambient air temperature, pressure, and humidity were digitized, recorded every 3 min, and averaged on an hourly basis. The system calibration was carried out at every measurement. Energy expenditure $(\mathrm{J} / \mathrm{min})$ was calculated using Weir's formula (17): $\left(1.106 \times \mathrm{VCO}_{2}+3.941 \times \mathrm{VO}_{2}\right) \times 4.186$.

CT scanning. After the 6-wk intervention, fat mass in the visceral and subcutaneous regions was estimated by X-ray CT scan (Latheta LCT-100, Aloka, Tokyo, Japan). The tube voltage of the X-ray generator was $50 \mathrm{kV}(1 \mathrm{~mA})$. Scan time was $4.5 \mathrm{~s} / \mathrm{slice}$. Rats (non-fasting) were anesthetized by intraperitoneal injection of sodium pentobarbital $(50 \mathrm{mg} / \mathrm{kg})$ and their body 
Table 2. Initial and final body weights, body weight gain, food intake, feed efficiency and serum adipo-cytokines concentrations in each group. ${ }^{1}$

\begin{tabular}{|c|c|c|c|c|c|c|c|}
\hline & \multirow{2}{*}{ LCT-C } & \multirow{2}{*}{ MCT-C } & \multirow{2}{*}{ LCT-E } & \multirow{2}{*}{ MCT-E } & \multicolumn{3}{|c|}{$p$-values ${ }^{2}$} \\
\hline & & & & & MCT & $\mathrm{Ex}^{4}$ & $\mathrm{MCT} \times \mathrm{Ex}$ \\
\hline Initial body weight (g) & $130.0 \pm 1.3$ & $129.8 \pm 1.4$ & $130.1 \pm 1.3$ & $130.1 \pm 1.3$ & 0.655 & 0.670 & 0.786 \\
\hline Final body weight (g) & $275.4 \pm 3.4$ & $267.2 \pm 3.9$ & $259.9 \pm 5.5^{\mathrm{a}}$ & $256.2 \pm 3.7^{\mathrm{b}}$ & 0.163 & 0.004 & 0.568 \\
\hline Body weight gain $(\mathrm{g} / 6 \mathrm{wk})$ & $135.8 \pm 4.1$ & $130.1 \pm 1.6^{\mathrm{a}}$ & $129.1 \pm 4.5^{\mathrm{a}}$ & $127.2 \pm 4.0$ & 0.230 & 0.001 & 0.506 \\
\hline Food Intake (g/6 wk) & $574.0 \pm 7.9$ & $584.2 \pm 5.9$ & $557.1 \pm 12.2$ & $567.7 \pm 9.4$ & 0.267 & 0.084 & 0.980 \\
\hline Feed Efficiency $(\%)^{3}$ & $25.2 \pm 0.3$ & $23.4 \pm 0.7^{\mathrm{a}}$ & $23.2 \pm 0.6^{\mathrm{a}}$ & $22.4 \pm 0.5$ & 0.034 & 0.050 & 0.467 \\
\hline Adiponectin $(\mu \mathrm{g} / \mathrm{L})$ & $3.27 \pm 0.3$ & $4.22 \pm 0.3^{\mathrm{a}}$ & $2.99 \pm 0.1$ & $4.12 \pm 0.3^{\mathrm{c}}$ & $<0.001$ & 0.242 & 0.989 \\
\hline Leptin $(\mu \mathrm{g} / \mathrm{L})$ & $3.77 \pm 0.4$ & $3.88 \pm 0.4$ & $2.46 \pm 0.3^{\mathrm{a}}$ & $1.78 \pm 0.1^{\mathrm{b}}$ & 0.140 & $<0.001$ & 0.260 \\
\hline
\end{tabular}

${ }^{1}$ Value are means \pm SE, $n=6$. ${ }^{a}$ Significantly different from LCT-C group; ${ }^{b}$ significantly different from MCT-C group;

${ }^{\mathrm{c}}$ significantly different from LCT-E group $(p<0.05)$.

${ }^{2}$ Significant main effects of MCT and Ex were determined by two-way ANOVA.

${ }^{3}$ Body weght gain $(\mathrm{g} / 6 \mathrm{wk}) /$ food intake $(\mathrm{g} / 6 \mathrm{wk}) \times 100(\%)$.

${ }^{4}$ Ex: exercise.
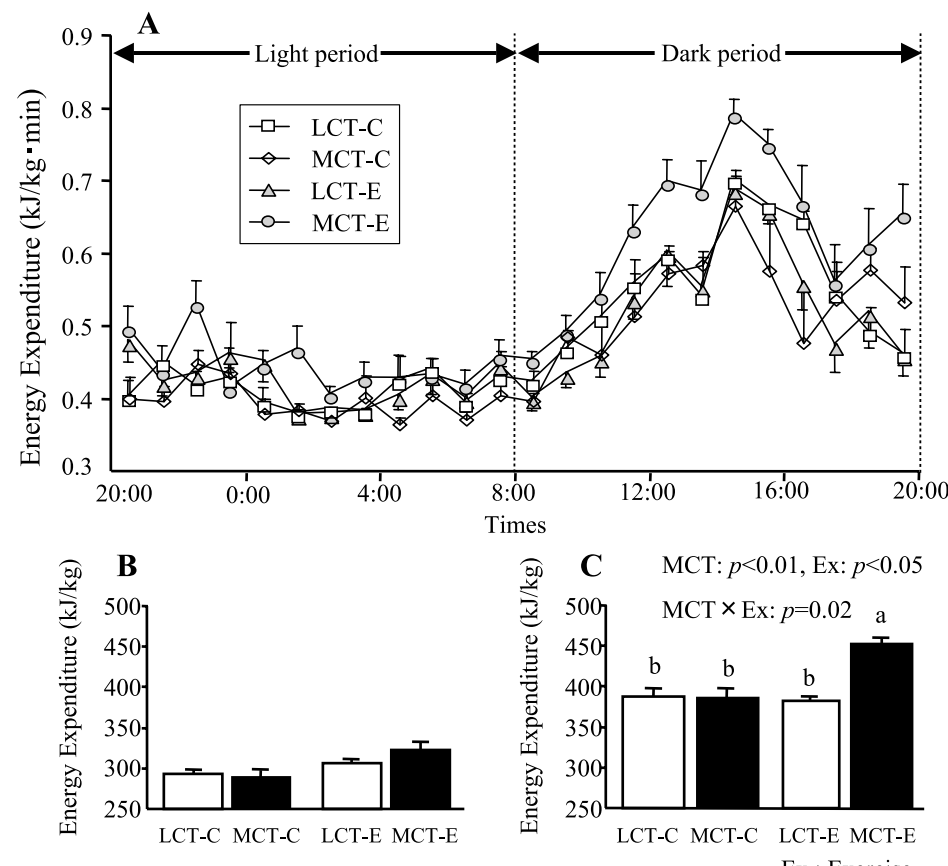

Fig. 2. Energy expenditure over $24 \mathrm{~h}$ measured by metabolic chamber. All of the rats were placed in the metabolic chamber for 24-h respiratory gas analysis. Oxygen consumption and carbon dioxide production were measured, and the energy expenditure was calculated from these values. (A) Data of energy expenditure over $24 \mathrm{~h}$ for each group. (B) Total of energy expenditure during the light period (20:00-8:00). (C) Total of energy expenditure during the dark period (8:00-20:00). Data are means \pm SE for 6 rats in each group. The main effects of MCT diet and exercise interventions, and their interaction were analyzed by two-way ANOVA. There were significant effects of MCT diet $(p<0.01)$, exercise $(p<0.05)$, and their interaction $(p=0.02)$. Bars with different letters are significantly different $(p<0.05)$.

weights were measured at 10:00. The anesthetized rats were placed in the CT scanner and their whole body was scanned along the body axis at $2-\mathrm{mm}$ intervals. Fat volumes were measured using this device according to the manufacturer's protocol (Latheta software version 1.00). Contiguous 2-mm slice images between the diaphragm and the bottom of the abdominal cavity were used for quantitation of visceral and subcutaneous fat volumes (Fig. 1A). CT-image was displayed at $40 \mathrm{~mm}$ below the diaphragm (Fig. 1B).

Survey of visceral fat mass by dissection. The rats which were CT scanned were euthanatized by exsanguination under anesthesia. Fat pads from three visceral regions, that is the epidydimal, perirenal (including retroperitoneal fat mass) and mesenteric (including omental fat mass), were carefully removed.

Serum adipo-cytokines. Serum was obtained by centrifuging at $300 \times g$ for $15 \mathrm{~min}$ at $4^{\circ} \mathrm{C}$ and frozen at $-20^{\circ} \mathrm{C}$. The serum adiponectin and leptin concentrations were measured by ELISA kits (Otsuka, Tokyo, and Morinaga, Yokohama, Japan, respectively) in accordance with the manufacturers' instructions. For the 


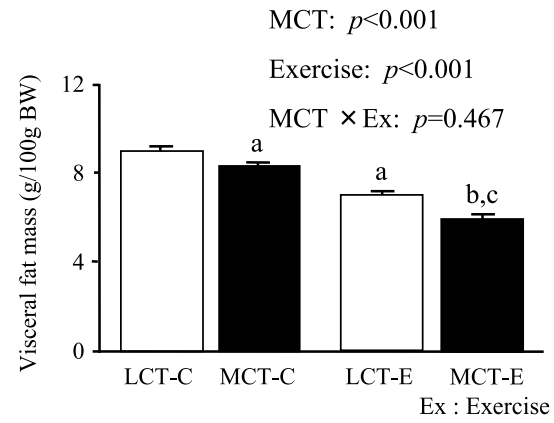

Fig. 3. CT scan data of visceral fat mass. Fat mass in the visceral region was estimated by X-ray CT scan. Data are means \pm SE for 6 rats in each group. The main effects of MCT diet and exercise, and their interaction were analyzed by two-way ANOVA. There were significant effects of MCT diet $(p<0.001)$, and exercise interventions $(p<0.001)$. There was no significant interaction between the two interventions. Bars with different letters are significantly different $(p<0.05)$. a, Significantly different from LCT-C group; b, significantly different from MCT-C group; c, significantly different from LCT-E group $(p<0.05)$.

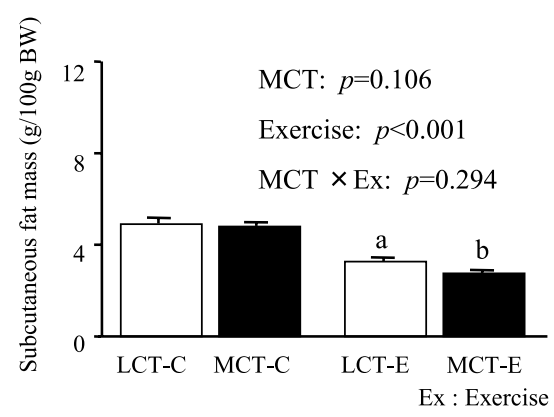

Fig. 4. CT scan data of subcutaneous fat mass. The fat mass in the subcutaneous region was estimated by Xray CT scan. Data are means \pm SE for 6 rats in each group. The main effects of MCT diet and exercise, and their interaction were analyzed by two-way ANOVA. There was a significant effect of exercise intervention $(p<0.001)$. There was no significant interaction between the MCT diet and exercise intervention. Bars with different letters are significantly different $(p<0.05)$. a, Significantly different from LCT-C group; $b$, significantly different from MCT-C group $(p<0.05)$.

absorbance measurement, we used a plate reader (Power Wave X340; Bio-Tek Instruments, Vermont, USA).

Statistical analysis. Data are expressed as means \pm SE. The main effects of the MCT diet and exercise, and interaction of the two interventions, were analyzed by two-way ANOVA (SPSS version 13-J; SPSS, Tokyo, Japan). When the significant interaction effect was detected, the significance of differences among the different groups were determined by one-way ANOVA followed by Fisher's protected least-significant-difference test. Pearson correlation was used to determine associations between CT scan and dissection data. Differences were considered significant at a level of $p<0.05$.
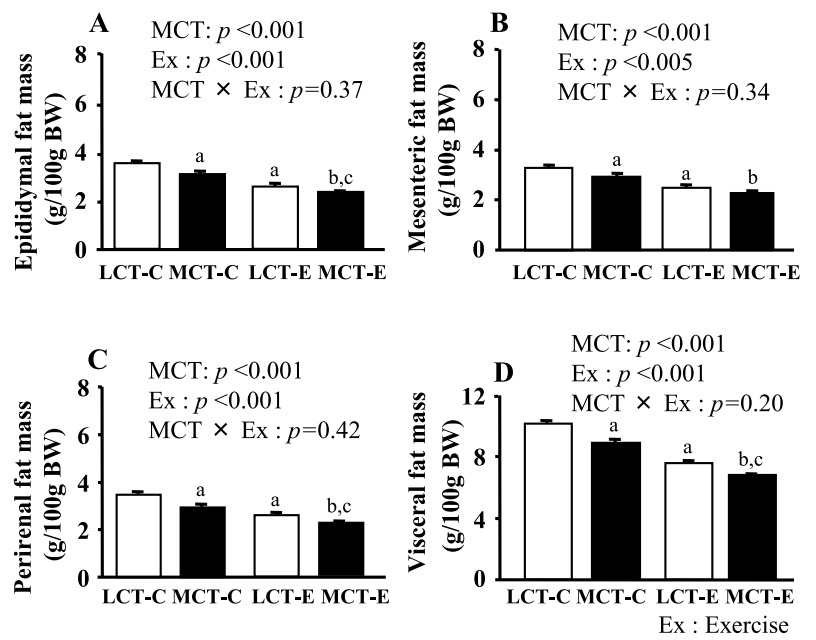

Fig. 5. Dissection data of visceral fat mass. (A) Epididymal fat mass, (B) mesenteric fat mass, (C) perirenal fat mass, and (D) visceral fat mass (the sum of the regions of the epididymal, mesenteric, and perirenal fat mass). Rats were divided into LCT-C, MCT-C, LCT-E, and MCTE groups. Data are means \pm SE for 6 rats in each group. The main effects of MCT diet and exercise, and their interaction were analyzed by two-way ANOVA. There was a significant effect of MCT diet on the each region's, and total visceral fat mass (epididymal, $p<0.001$; mesenteric, $p<0.005$; perirenal, $p<0.001$; visceral, $p<0.001)$. There was a significant effect of exercise on the each region's, and total visceral fat mass (epididymal, $p<0.001$; mesenteric, $p<0.001$; perirenal, $p<0.001$; visceral, $p<0.001$ ). There was no significant interaction between the MCT diet and exercise. Bars with different letters are significantly different $(p<0.05)$. a, Significantly different from LCT-C group; $\mathrm{b}$, significantly different from MCT-C group; c, significantly different from LCT-E group $(p<0.05)$.

\section{RESULTS}

\section{Food intake and body mass}

Food intake did not differ among the four groups. Food efficiency in LCT-C rats was greater than the other three groups (Table 2). The final body weight and body weight gain were significantly lower in the exercise groups (LCT-E, MCT-E) than the control groups (LCT-C, MCT-C).

Energy expenditure over $24 \mathrm{~h}$

Each group tended to exhibit higher energy expenditure during the dark period (08:00-20:00) than in the light period (20:00-08:00). Significant effects of the MCT diet $(p<0.05)$ and exercise $(p<0.05)$ can be seen in the increase of the energy expenditure during the dark period. There was also significant $(p=0.017)$ interaction between the MCT diet and exercise. The MCT-E group exhibited significantly $(p<0.05)$ higher energy expenditure than the other three groups (Fig. 2).

Effects of MCT diet and exercise on fat mass measured by CT scan

Significant effects of the MCT diet $(p<0.001)$ and exercise $(p<0.001)$ can be seen in the reduction of visceral fat mass (Fig. 3). The value of visceral fat was lower in the MCT-C group than in the LCT-C group. 


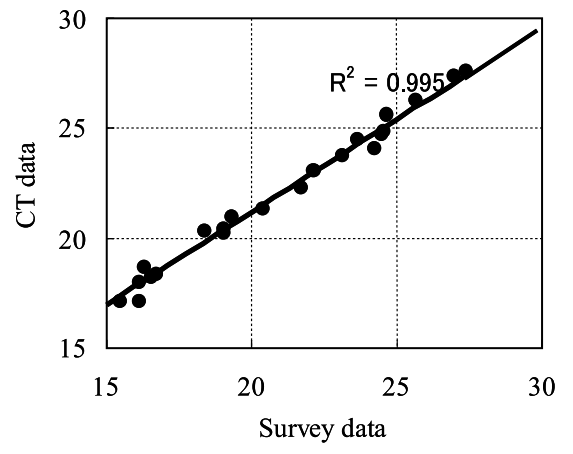

Fig. 6. Linearity of CT data and survey data. The correlation coefficients (Pearson's product-moment correlation coefficient) of the visceral fat mass data were obtained by both CT $(n=24)$ and dissection $(n=24)$.

Furthermore, the visceral fat was lower in the MCT-E group than in either group alone. There was a significant effect of exercise $(p<0.001)$ on the reduction of subcutaneous fat mass (Fig. 4). However, no interaction was observed between the MCT diet and exercise interventions.

Effects of MCT diet and exercise on fat mass measured by dissection

The visceral fat mass, which includes the epididymal (Fig. 5A), mesenteric (Fig. 5B), and perirenal (Fig. 5C) regions, were measured. There were significant effects of exercise on each region (epididymal, $p<0.001$; mesenteric, $p<0.001$; perirenal, $p<0.001)$. Similarly, there were significant effects of the MCT diet on each region (epididymal, $p<0.001$; mesenteric, $p<0.005$; perirenal, $p<0.001$ ). In addition, there were significant effects of the MCT diet $(p<0.001)$ and exercise intervention $(p<0.001)$ on reduction of the visceral fat mass (Fig. 5D). However, there was no significant interaction between the MCT diet and exercise interventions.

Correlations between the visceral fat measured by CT scan and dissection

The visceral fat mass obtained by CT scan (Fig. 3) and by dissection (sum of epididymal, mesenteric and perirenal regions, Fig. 5D) were significantly correlated $(r=0.995, p<0.001)$ (Fig. 6).

Serum adipo-cytokines data

The serum adiponectin concentration was higher in the MCT diet groups than in the LCT diet groups. However, the serum leptin concentration was lower in the exercise groups than in the control groups (Table 2).

\section{DISCUSSION}

The novel findings of this study are as follows: 1) Compared with the LCT control diet in rats, the MCT diet reduced the accumulation of visceral fat mass; 2) The combined intervention of MCT diet and exercise showed an additive effect on reduction of fat mass accumulation, and this effect may be related to increased energy expenditure.

It is known that, compared with LCT diets, an MCT has a faster rate of oxidation that can increase the energy expenditure and diet-induced thermogenesis (DIT) (18). These biological characteristics of MCT may contribute to fat-lowering effects. On the other hand, it has been documented that exercise training can enhance the lipolysis of adipose tissue by increasing fat oxidation and fatty acid availability $(13,14)$. Based on the efficacy of each MCT diet and exercise training, we hypothesized that combined intervention would result in maximizing the potential for preventing accumulation of fat mass. As we expected, a pronounced and additive effect of the two interventions was observed. We presume that exercise can enhance the $\beta$-oxidation and availability of MCFA. Furthermore, it is likely that the exercise may increase lipolysis of adipose, mediated by $\beta$-adrenergic stimulation (19). In our study, we estimated 24-h energy expenditure, which is considered relevant to body composition. The combined interventions of MCT diet and exercise significantly enhanced the energy expenditure during the dark period (active period), but not during the light period. However, either intervention alone did not affect the energy expenditure. This result does not sufficiently reflect our body fat accumulation data, and therefore a future study should be designed to clarify the relationship between energy expenditure and fat mass accumulation.

Adipose tissue not only stores excess energy in the form of fat, but also releases physiologically active mediators known as adipo-cytokines, such as leptin and adiponectin (20). Recently, the adipo-cytokines have demonstrated links between obesity and insulin resistance in rodents and humans $(21,22)$. In this study, we found a significant effect of MCT diet on the increase of serum adiponectin levels. However, exercise had no effect on serum adiponectin levels. This result is similar to Bhattacharya et al.'s (23) findings which reported that exercise alone did not increase serum adiponectin levels. In a previous study (24), a rise of serum adiponectin level by MCT diet was confirmed. In that study, mRNA levels related to adiponectin gene expression were also raised. Retinoid X receptor (RXR) and peroxisome proliferators-activated receptor $\gamma(\operatorname{PPAR} \gamma)$ mRNA levels were significantly higher in the rats fed with MCT. These results suggest that improved glucose tolerance in rats fed with MCT may be ascribed to higher serum adiponectin level (24). Regarding the leptin, the significant main effect on decreasing the circulating level was found in exercise intervention only. However, the combined intervention of MCT diet and exercise showed a trend for a greater effect on regulation of serum adipocytokines than either one alone.

Recently, dual-energy X-ray absorptiometry (DXA) and X-ray computed tomography (CT) scan have been used for determining in vivo body composition in humans, as well as in experimental animals (25). In this study, we found a linear relationship of the visceral fat mass measured between CT scan and dissection. Nagy and Clair (26) reported that DXA-derived data tend to overestimate carcass fat mass in rat. Instead of using the DXA (2-Dimension), we tried the CT-scan (3Dimension) for estimating fat mass in this study. Because every contiguous 2-mm CT-scanned slice was estimated in the abdominal region, fat mass was not 
overestimated. Therefore, we suggest that the CT-scan can be used to more accurately predict body composition.

In conclusion, we observed that combined intervention employing MCT diet and exercise had an additive effect in preventing body fat accumulation, especially in the visceral region, and that these effects may be related to increased energy expenditure. Further studies are necessary to define the detailed mechanism of interaction between exercise and MCT diet to reduce visceral adipose tissue.

\section{Acknowledgments}

We would like to thank Dr. Hisami Shinohara, Mr. Keiichi Kojima, and Ms. Akiko Ogawa for their technical assistance. We are grateful to Ms. Kaori Nakajima for her helpful discussions in preparation of the manuscript. We are also grateful to Dr. Michihiro Sugano for his valuable advice in revision of the manuscript.

\section{REFERENCES}

1) Kaunitz H, Slanetz CA, Johnson RE, Babavan VK, Barsky G. 1958. Nutritional properties of the triglycerides of saturated fatty acids of medium chain-length. J Am Oil Chemists Soc 35: 10-13.

2) Kaunitz H, Slanetz CA, Johnson RE, Babayan VK, Barsky G. 1958. Relation of saturated, medium- and longchain triglycerides to growth, appetite, thirst and weight maintenance requirements. J Nutr 64: 513524.

3) Fureund G, Weinsier RL. 1966. Standardized ketosis in man following medium chain triglyceride ingestion. Metabolism 15: 980-991.

4) Bach AC, Babayan VK. 1982. Medium-chain triglycerides: an update. Am J Clin Nutr 36: 950-962.

5) Noguchi O, Shinohara H, Kubota F, Tsuji H, Aoyama T. 2002. Nutritional effects of randomly interesterified and physically mixed oils containing medium-chain fatty acids on rats. J Oleo Sci 51: 699-703.

6) Kasai M, Nosaka N, Maki H, Negishi S, Aoyama T, Nakamura M, Suzuki Y, Tsuji H, Uto H, Okazaki M, Igarashi O, Kondo K. 2003. Effect of dietary medium- and long-chain triacylglycerols (MLCT) on accumulation of body fat in healthy humans. Asia Pac J Clin Nutr 12: 151-160.

7) Despres JP, Pouliot MJ, Moorjani S, Nadeau A, Tremblay A, Lupien PJ, Theriault G, Bouchard C. 1991. Loss of abdominal fat and metabolic response to exercise training in obese women. Am J Physiol 261: E159-E167.

8) Fujioka S, Matsuzawa Y, Tokunaga K, Kawamoto T, Kobatake T, Keno Y, Kotani K, Yoshida S, Tarui S. 1991. Improvement of glucose and lipid metabolism associated with selective reduction of intra-abdominal visceral fat in premenopausal women with visceral fat obesity. Int $J$ Obes 15: 853-859.

9) Williams MJ, Hunter GR, Kekes-Szabo T, Snyder S, Treuth MS. 1997. Regional fat distribution in women and risk of cardiovascular disease. Am J Clin Nutr 65: 855-860.

10) Despres JP, Tremblay A, Nadeau A, Bouchard C. 1988. Physical training and changes in regional adipose tissue distribution. Acta Med Scand Suppl 723: 205-212.

11) Wilmore JH. 1996. Increasing physical activity: alterations in body mass and composition. Am J Clin Nutr
63: $456 \mathrm{~S}-460 \mathrm{~S}$.

12) Despres JP, Lamarche B. 1993. Effects of diet and physical activity on adiposity and body fat distribution: implication for the prevention of cardiovascular disease. Nutr Res Rev 6: 137-159.

13) Bukowiecki L, Lupien J, Follea N. 1980. Mechanism of enhanced lipolysis in adipose tissue of exercise-trained rats. Am J Physiol 239: E422-E429.

14) Crampes F, Beauville M, Riviere D, Garrigues M. 1986. Effect of physical training in humans on the response of isolated fat cells to epinephrine. J Appl Physiol 61: 2529.

15) Fushiki T, Matsumoto K, Inoue K, Kawada T, Sugimoto E. 1995. Swimming endurance capacity of mice is increased by chronic consumption of medium-chain triglycerides. J Nutr 125: 531-539.

16) Reeves PG, Nielsen FH, Fahey GC Jr. 1993. AIN-93 purified diets for laboratory rodents: final report of the American Institute of Nutrition ad hoc writing committee on the reformulation of the AIN-76A rodent diet. $J$ Nutr 123: 1939-1951.

17) Weir JBV. 1949. New methods for calculating metabolic rate with special reference to protein metabolism. J Physiol 109: 1-9.

18) Noguchi O, Takeuchi H, Kubota F, Tsuji H, Aoyama T. 2002. Larger diet-induced thermogenesis and less body fat accumulation in rats fed medium-chain triacylglycerols than in those fed long-chain triacylglycerols. $J$ Nutr Sci Vitaminol 48: 524-529.

19) Horowitz JF, Klein S. 2000. Lipid metabolism during endurance exercise. Am J Clin Nutr 72: 558S-563S.

20) Matsuzawa Y, Funahashi T, Nakamura T. 1999. Molecular mechanism of metabolic syndrome X: contribution of adipocytokines adipocyte-derived bioactive substances. Ann NY Acad Sci 892: 146-154.

21) Nagao K, Inoue N, Wang YM, Yanagita T. 2003. Conjugated linoleic acid enhances plasma adiponectin level and alleviates hyperinsulinemia and hypertension in Zucker diabetic fatty (fa/fa) rats. Biochem Biophys Res Commun 310: 562-566.

22) Bruun JM, Lihn AS, Verdich C, Pedersen SB, Toubro S, Astrup A, Richelsen B. 2003. Regulation of adiponectin by adipose tissue-derived cytokines: in vivo and in vitro investigations in humans. Am J Physiol Endocrinol Metab 285: E527-E533.

23) Bhattacharya A, Rahman MM, Sun D, Lawrence R, Mejia W, McCarter R, O'Shea M, Fernandes G. 2005. The combination of dietary conjugated linoleic acid and treadmill exercise lowers gain in body fat mass and enhances lean body mass in high fat-fed male Balb/C mice. J Nutr 135: 1124-1130.

24) Takeuchi H, Noguchi O, Sekine S, Kobayashi A, Aoyama T. 2006. Lower weight gain and higher expression and blood levels of adiponectin in rats fed medium-chain TAG compared with long-chain TAG. Lipids 41: 207212.

25) Fan W, Yanase T, Nomura M, Okabe T, Goto K, Sato T, Kawano H, Kato S, Nawata H. 2005. Androgen receptor null male mice develop late-onset obesity caused by decreased energy expenditure and lipolytic activity but show normal insulin sensitivity with high adiponectin secretion. Diabetes 54: 1000-1008.

26) Nagy TR, Clair AL. 2000. Precision and accuracy of dual-energy X-ray absorptiometry for determining in vivo body composition of mice. Obes Res 8: 392-398. 